\title{
Primary Steps of pH-Dependent Insulin Aggregation Kinetics are Governed by Conformational Flexibility
}

\author{
Jürgen Haas, ${ }^{[\mathrm{a}]}$ Esteban Vöhringer-Martinez ${ }_{1}^{[\mathrm{a}]}$ Andreas Bögehold, ${ }^{[\mathrm{b}]}$ Dirk Matthes, ${ }^{[\mathrm{a}]}$ \\ Ulf Hensen, ${ }^{[a]}$ Avishay Pelah, ${ }^{[c]}$ Bernd Abel, $^{[b]}$ and Helmut Grubmüller ${ }^{*[a]}$
}

\begin{abstract}
Insulin aggregation critically depends on $\mathrm{pH}$. The underlying energetic and structural determinants are, however, unknown. Here, we measure the kinetics of the primary aggregation steps of the insulin monomer in vitro and relate it to its conformational flexibility. To assess these primary steps the monomer concentration was monitored by mass spectrometry at various $\mathrm{pH}$ values and aggregation products were imaged by atomic force microscopy. Lowering the $\mathrm{pH}$ from 3 to 1.6 markedly accelerated the observed aggregation kinetics. The influence of $\mathrm{pH}$ on the monomer structure and dynamics in solution was studied by molecular dynamics simulations, with the
\end{abstract}

protonation states of the titrable groups obtained from electrostatic calculations. Reduced flexibility was observed for low $\mathrm{pH}$ values, mainly in the $\mathrm{C}$ terminus and in the helix of the $B$ chain; these corresponded to an estimated entropy loss of $150 \mathrm{Jmol}^{-1} \mathrm{~K}^{-1}$. The striking correlation between entropy loss and $\mathrm{pH}$ value is consistent with the observed kinetic traces. In analogy to the well-known $\Phi$ value analysis, this result allows the extraction of structural information about the rate determining transition state of the primary aggregation steps. In particular, we suggest that the residues in the helix of the $B$ chain are involved in this transition state.

\section{Introduction}

Insulin regulates the uptake and storage of glucose in the liver and muscles as well as the biosynthesis of triglycerides by fat cells. It is produced in the $\beta$-cells of the islets of Langerhans in the pancreas and is stored there as zinc-ligated hexamer microcrystals in acidified secretory granules. ${ }^{[1,2]}$ Upon release into the blood serum, and triggered by the accompanying $\mathrm{pH}$ change, the hexamer dissociates into three dimers and then subsequently into monomers, ${ }^{[3]}$ which is its physiologically active form.

Disorders in human insulin metabolism can cause type II diabetes, a disease affecting a large share of the population. A notorious complication for intravenous insulin injection and subcutaneous infusion is the pathogenic deposition of the protein at the injection sites due to aggregation at high local concentration. ${ }^{[4,5]}$ Moreover, insulin aggregation still poses a major challenge for pharmaceutical production and clinical formulation. ${ }^{[6,7]}$ Accordingly, insights into this deposition process can facilitate the development of improved insulin variants.

A particular type of aggregation is the formation of amyloid fibrils, observed, for example, in Alzheimer's disease ${ }^{[8]}$ or in the degradation of protein-based drugs. ${ }^{[9]}$ The transition to well-organized fibrils with characteristic cross- $\beta$ sheet motif has also been observed at low $\mathrm{pH}$ for insulin under various conditions; ${ }^{[10-19]}$ this renders insulin a well-defined prototypic system for the study of fibril formation, in vitro. Bovine insulin, in particular, which differs from the human protein at three amino acid positions, has been shown to aggregate faster compared to the human variant. ${ }^{[20]}$ Despite several investigations at lower $\mathrm{pH}$ values ${ }^{[20,21]}$ no systematic study of the primary aggregation steps as a function of $\mathrm{pH}$ has been reported. Moreover, the insoluble and uncrystallizable nature of amyloid fibrils still limits the access to structural high-resolution data. Recently, two short segments of the human insulin sequence have been identified to form amyloid-like fibrils as well as microcrystals, which might help to elucidate structural details of the aggregation process. ${ }^{[22,23]}$

The exact mechanism of insulin amyloid fibril formation, however, remains unresolved. ${ }^{[11,12,24]}$ Despite the vast number of studies about fibrils and aggregation intermediates, only little is known about the primary steps of the aggregation. ${ }^{[17,20]}$ The main reason for this slow progress in most experimental as well as theoretical approaches is related to the fact that the aggregation mechanism consists of multiple steps and that the rate-limiting transition state is still unknown.

It is likely that the titrable groups of insulin play a crucial role in these primary steps (Figure 1). At its isoelectric point of

[a] Dr. J. Haas, ${ }^{+}$Dr. E. Vöhringer-Martinez, ${ }^{+}$D. Matthes, Dr. U. Hensen, Prof. Dr. H. Grubmüller

Department of Theoretical and Computational Biophysics Max-Planck-Institute for Biophysical Chemistry

Am Fassberg 11, 37077 Göttingen (Germany)

Fax: (+49) 551-2012302

E-mail:hgrubmu@gwdg.de

[b] Dr. A. Bögehold, Prof. Dr. B. Abel

Wilhelm-Oswald-Institut für Physikalische und Theoretische Chemie University Leipzig, Linne-Strasse 2, 04103 Leipzig (Germany)

[c] Dr. A. Pelah

Laboratory of Cellular Dynamics

Max Planck Institute for Biophysical Chemistry

Am Fassberg 11, 37077 Göttingen (Germany)

$\left[{ }^{+}\right]$These authors contributed equally to this work.

Supporting information for this article is available on the WWW under http://dx.doi.org/10.1002/cbic.200900266. 


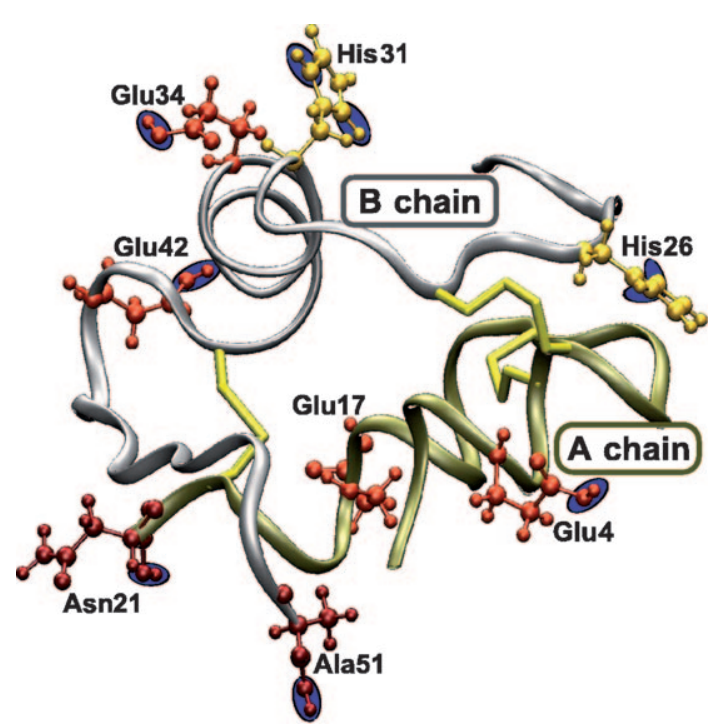

Figure 1. Crystal structure of insulin displaying the $A$ chain (21 residues) containing an intrachain disulfide bond, and the $B$ chain (30 residues) with two additional disulfide bonds linking the chains (PDB ID: 2BN3). The titrable groups in the $\mathrm{pH}$ range of 1 to 7 are highlighted with blue circles. The C-terminal regions are shown in dark red $\left(C_{\text {TER }}\right)$, glutamates are depicted in light red and histidines in yellow.

$\mathrm{pH}$ 5.3, insulin is neutral, and one might expect that protonation at lower $\mathrm{pH}$ results in charged monomers. This effect would slow down the first aggregation steps of the monomers via long range electrostatic repulsion. In addition, depending on the involved residues, protonation at low $\mathrm{pH}$ alters the hydrophilicity of the protein surface, which in turn would also affect the aggregation kinetics. ${ }^{[20]}$ An entropic effect on the dimerization process was suggested from theoretical studies. Zoete et al. have estimated the insulin dimerization binding free energy ${ }^{[25]}$ based on the crystal structure of the dimer. ${ }^{[26]}$ Calculation of the entropic contribution to this binding free energy revealed a loss in translational and rotational entropy as a dominant factor that is not overcome by the gain in vibrational entropy, which was estimated by normal mode analysis. ${ }^{[27]}$ The main entropic contribution, however, is expected from the protonation of titrable groups, which might affect the atomic fluctuations of a protein. Depending on how much these fluctuations are altered in any transition state between monomers and oligomers, the first steps of the aggregation process might also be influenced entropically.

In all three cases, an influence of $\mathrm{pH}$ on the primary aggregation steps of insulin is expected. If entropy plays a major role, structural information of the transition state can be extracted from the measured aggregation kinetics. Furthermore, any correlation of the monomer decay with the primary steps of aggregation could imply an early rather than a late transition state in the insulin aggregation kinetics, which eventually leads to fibrils.

Here, we test these hypotheses by a combined experimental and computational approach. The $\mathrm{pH}$-dependent aggregation is addressed by monitoring the monomer concentration of bovine insulin by mass spectrometry, while the $\mathrm{pH}$-dependent entropies of the monomer are calculated from computer simulations.

\section{Results}

\section{Aggregation kinetics}

The influence of $\mathrm{pH}$ value on the aggregation rate of the insulin monomer was investigated with time-resolved liquid beam (laser) desorption mass spectrometry by monitoring the decrease of insulin monomer abundance in aqueous solution as a function of time. The experiments were carried out at near ambient temperature $(313 \mathrm{~K})$ and between $\mathrm{pH} 1.6$ and 3 with typical insulin concentrations of $50 \mu \mathrm{M}$. The obtained concentration profiles are characterized by sigmodial time traces (Figure $2 \mathrm{~A}$ ) and a lag time $\tau$, which can be quantified by the time between the start of the kinetics and the time of half decay. ${ }^{[28,29]}$ This lag time as a function of $\mathrm{pH}$ can be observed
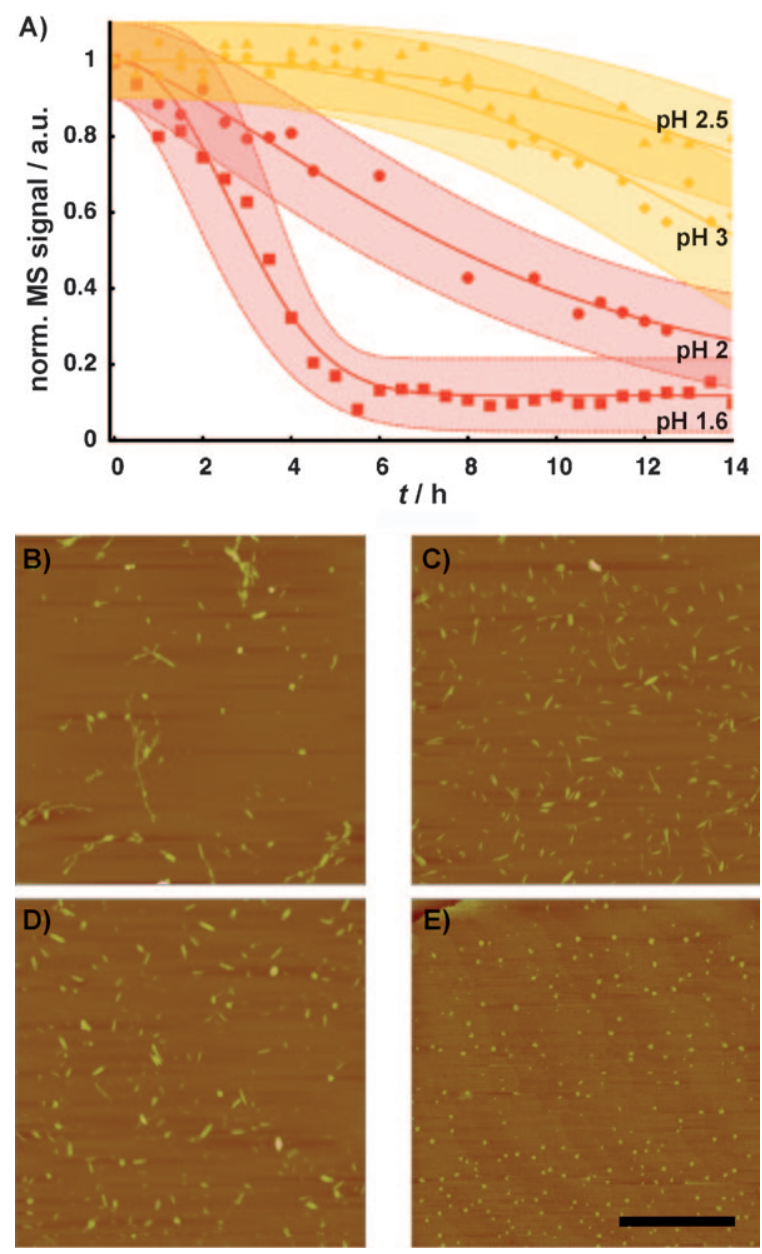

Figure 2. A) Relative intensities of the insulin monomer MS peak versus time. Intensities were measured relative to the mass peak intensity of an unaggregated protein sample at the corresponding $\mathrm{pH}$ value. The data points were fitted with a sigmodial function. The transparent bands around the time-resolved data points in the traces reflect the overall experimental uncertainties. Atomic force microscope images of the aggregated samples after $72 \mathrm{~h}$ at $\mathrm{pH}$ : B) $1.6, \mathrm{C}) 2.0$, D) 2.5, E) 3.0. The black bar corresponds to a length scale of $1 \mu \mathrm{m}$. 
in the time-resolved experiments. Significant populations of oligomers at higher masses have not been observed. For the employed experimental conditions, we verified that the monomer is the major species in the system using static light scattering, and calculated the equilibrium constant for dimerization as a function of $\mathrm{pH}$ (see the Supporting Information). Changes in monomer concentration due to a shift of the equilibrium cannot account for the $\mathrm{pH}$-dependent differences observed in the kinetics since the dependence of the kinetics on insulin monomer concentration has been found to be very small in the considered concentration range.

The measured kinetics depicted in Figure $2 \mathrm{~A}$ display the smallest lag time at the lowest $\mathrm{pH}$ of 1.6 and increased $\tau$ values by at least a factor of three at higher $\mathrm{pH}$ values. This $\mathrm{pH}$-dependence is consistent with data obtained at a higher temperature $(T=358 \mathrm{~K})$ by Nielsen et al., ${ }^{[20]}$ who followed the kinetics of fibril formation by means of thioflavin fluorescence.

Subsequent to the kinetic experiments the samples were cooled to $283-288 \mathrm{~K}$, which terminated the aggregation process. To monitor the formation of short fibrils, spherical protofibrils, and, in particular, to verify that this process is correlated with the monomer-decay kinetics, the aggregation products were imaged with atomic force microscopy (AFM). Indeed, the images and overall features agree with the results obtained by Jansen et al. ${ }^{[18]}$ The morphology of the observed aggregation products appeared to depend on the $\mathrm{pH}$ of the system (Figure $2 \mathrm{~B}$ ). In the $\mathrm{pH}$ range of 1.6 to 2.5 the products consisted of short, rod-like aggregates, several longer fibrils and a few fairly disordered aggregates. In contrast, at $\mathrm{pH} 3$, at which also rather slow kinetics was measured, only spherical aggregation species were observed. The more pronounced fibril structures for fast aggregation and the spherical nonfibrillar structures for slow overall kinetics suggest that in all cases a similar pathway, but different intermediates on the way to larger fibrils, were observed. The difference in the experiments appears to be the timescale of the overall aggregation kinetics and the stage of the aggregation when it was monitored with AFM.

Since lowering the $\mathrm{pH}$ of the solution is accompanied with successive protonation of titrable amino acids and in turn with an increase of the overall charge of the monomers, it might at first sight appear counterintuitive that a lower $\mathrm{pH}$ correlates with faster aggregation. The higher charge should increase the electrostatic interaction, that is, the repulsion between equally charged species, which is expected to slow down an encounter and in turn the aggregation.

To assess the influence of this electrostatic interaction, which would be reflected in a large contribution of the activation enthalpy to the total activation free energy, aggregation kinetics were also measured at a temperature of $358 \mathrm{~K}$ and $\mathrm{pH} 2$ (see the Supporting Information). Assuming an Eyring transition state model in combination with an Arrhenius behavior in this temperature range, ${ }^{[30]}$ these measurements yield a free energy activation barrier with entropic and enthalpic contributions that are in the same order of magnitude. At high $\mathrm{pH}$ and reduced charges on the monomers, a correspondingly reduced enthalpic contribution is expected, and, therefore, the total activation free energy barrier would include a larger entropic part.
These results assign the entropy an important role in the aggregation kinetics. Especially at around $\mathrm{pH} 2$ small changes in $\mathrm{pH}$ result in pronounced changes in observed kinetics.

\section{pH-dependent flexibility}

In order to understand this result and to assess the influence of $\mathrm{pH}$ on the internal dynamics, energetics, and entropies of the insulin monomer, extensive all-atom MD simulations were carried out at $\mathrm{pH}$ values ranging from 1 to 7 . Upon changing the $\mathrm{pH}$ in this range, the protonation sites will be deprotonated in the sequence: carboxyl groups of the $C$ termini $\left(C_{\text {TER }}\right)$, carboxyl groups of the glutamates $(\mathrm{E})$, and the imidazole nitrogens of the histidines $(\mathrm{H}$; Figure 1$)$. Four different static protonation states of the protonable groups in the monomer were considered, and the respective $\mathrm{pH}$ was derived from $\mathrm{p} K_{\mathrm{a}}$ values obtained from continuum electrostatic calculations (see the Supporting Information). ${ }^{[31]}$

All titrable groups were found to be protonated at $\mathrm{pH}<1$. Correspondingly, system $\mathrm{HEC}_{\text {TER }}$ was set up with doubly protonated histidines, glutamates and $\mathrm{C}$ termini to describe this $\mathrm{pH}$ range. Similarly, for $1<\mathrm{pH}<2$ (system $\mathrm{HE}$ ) histidines (doubly) and glutamates were protonated; for $2<\mathrm{pH}<5$ (system $\mathrm{H}$ ) all histidines were doubly protonated; and from $\mathrm{pH}>5$ (system 0 ) one of the two nitrogens in each histidine was deprotonated. Accordingly, systems $\mathrm{HE}$ and $\mathrm{H}$ correspond to the measured kinetics at $\mathrm{pH}$ values below or above $\mathrm{pH} 2$, respectively. All four systems were each simulated for $95 \mathrm{~ns}$ at $313 \mathrm{~K}$.

Figure $3 \mathrm{~A}$ shows the four ensembles obtained. System $\mathrm{H}$ displays an increased flexibility of the C-terminal B chain; this is in agreement with previously reported experimental and theoretical studies. ${ }^{[2,5,32]}$ The secondary structure of the insulin monomer in all systems is overall preserved, except for system $\mathrm{H}$, in which initial unfolding events in the first helix of the $A$ chain and the helix of the $B$ chain are observed. This is in agreement with a previously reported increase in $\Delta G_{\text {unfolding }}$ in ${ }^{1} H$ NMR spectroscopy titrations of human insulin. ${ }^{[2,12]}$ For a $\mathrm{pH}$ between 1 and 2 (system HE) an unexpectedly reduced flexibility was observed.

The differential flexibility is largely due to competing $\mathrm{pH}$ dependent interactions between the three pairwise contacts formed by the $C$ terminus of the B chain (Ala51) and the N-terminal residues of the $A$ chain (Gly1 and Glu4). Due to steric restraints, only two of these three contacts are formed at a time. When all titrable groups, including Ala51, are protonated, and Glu4 and Ala51 are neutral (system $\mathrm{HEC}_{\text {TER }}$ ), the interactions between all three pairs are equally weak, which implies frequent competitive contact-pair formation and rupture; this explains the observed high flexibility (see the Supporting Information). In contrast, for system HE, Ala51 is deprotonated, and one single, strong salt bridge is formed between Gly 1 and Ala51; this effectively locks the $C$ terminus of the $B$ chain. For the other two systems ( $\mathrm{H}$ and 0 ) Glu4 is also deprotonated; this allows for the formation of two competing salt bridges to Gly1. This results in a strong competitive interaction between Gly1, Glu4, and Ala51, and explains the higher overall flexibility at $\mathrm{pH} 2-5$. Finally, at high $\mathrm{pH}$ the singly protonated histidines 
A)
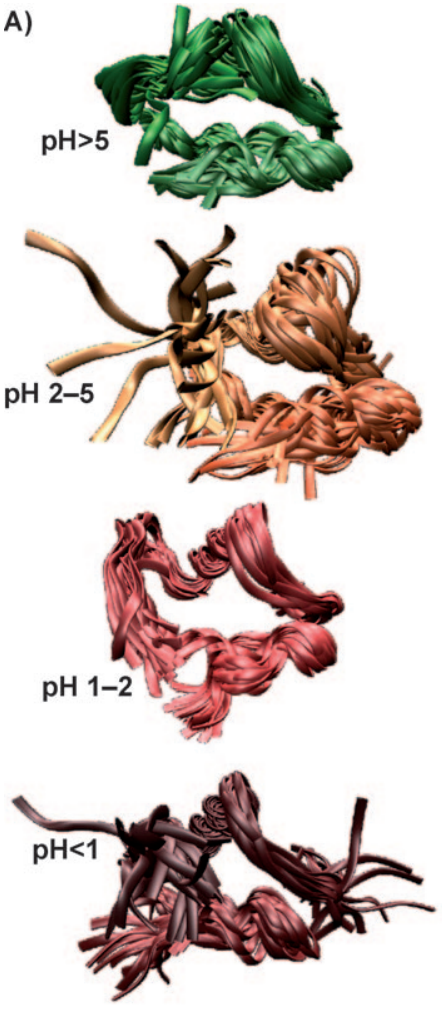

B)
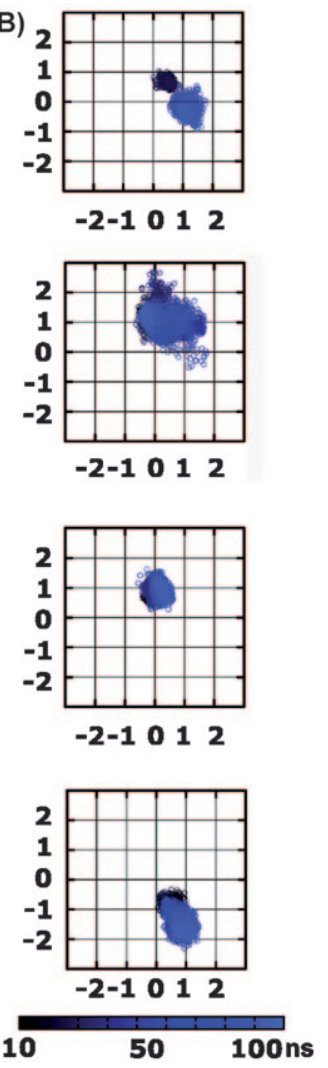

Figure 3. A) Illustration of the fluctuations of the four simulated systems. From top to bottom: $0(\mathrm{pH}>5), \mathrm{H}(\mathrm{pH} 2-5), \mathrm{HE}(\mathrm{pH} 1-2), \mathrm{HEC}_{\text {TER }}(\mathrm{pH}<1)$. B) Comparison of the respective conformational space sampled (data shown in $\mathrm{nm}$ ). The individual trajectories were projected onto the two most prominent, global eigenmodes, which were obtained from a principal component analysis (PCA) over the $C_{\alpha}$ atoms of the combined trajectories of the four systems.

allow for interactions between Ala51 and Ser30; this effectively locks the $\mathrm{C}$ terminus again, albeit at a different position, and is in agreement with NMR spectroscopy data. ${ }^{[2]}$

The flexibility of each system is quantified by a principal component analysis (PCA). The projection of the four trajectories onto the common essential subspace spanned by the first two eigenvectors with the largest eigenvalues (Figure 3B) describes those dominant collective motions of the protein that contribute most to the atomic fluctuations observed in the simulations. The area covered represents the extent of the explored conformational space, and quantifies the reduced flexibility for system HE as compared to the other systems. Apparently, the largest fluctuations are observed for system $\mathrm{H}$.

\section{Entropy estimate}

The larger area seen for system $\mathrm{H}$ suggests a higher entropy. To quantify the conformational entropies of each system, a full correlation analysis (FCA $)^{[3,34]}$ in combination with a density estimate of the complete configurational space was carried out (see the Supporting Information). This entropy estimate, however, depends on how complete the configurational phase space density is sampled and, hence, on the simulation time.

Accordingly, estimates obtained from short trajectories tend to underestimate the entropy. To address this convergence issue, we calculated the individual entropies of the $C_{\alpha}$ atoms using trajectories of different lengths, which had been started from different points during the simulations.

Figure 4 displays the entropies of the four systems obtained as a function of the time length used for analysis. A significantly larger entropy $\left(\sim 150 \mathrm{~J} \mathrm{~mol}^{-1} \mathrm{~K}^{-1}\right)$ for all analysis time lengths was observed for system $\mathrm{H}$ compared to all other systems.

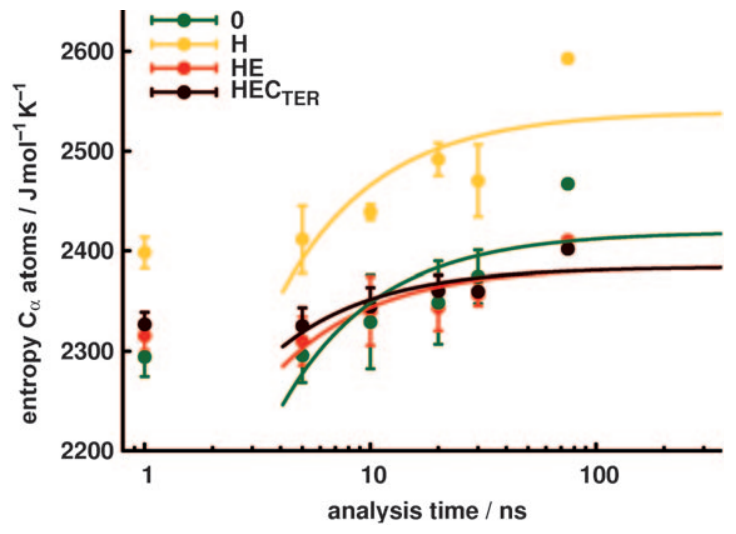

Figure 4. The FCA/density estimated entropies obtained for different trajectory lengths of the four simulated systems. The largest entropy was observed in the range of $\mathrm{pH} 2-5$ (system $\mathrm{H})$. From top to bottom: $0(\mathrm{pH}>5), \mathrm{H}$ $(\mathrm{pH} 2-5), \mathrm{HE}\left(\mathrm{pH} \mathrm{1-2)}, \mathrm{HEC}_{\text {TER }}(\mathrm{pH}<1)\right.$.

As a control, we also applied the established Schlitter method. ${ }^{[35,36]}$ Although this method relies on a (quasi-)harmonic approximation of the phase-space density and, therefore, does not account for nonlinear correlations, it has the advantage that it provides an upper bound for the entropy. Indeed, the entropies calculated with the FCA method were consistently smaller for all systems and, therefore, are assumed to be more accurate.

\section{Discussion}

The observed entropy difference between systems HE and $\mathrm{H}$, together with the faster, measured aggregation kinetics of the charged monomers at low $\mathrm{pH}$ and the temperature-dependent measurements at $\mathrm{pH} 2$ imply that entropy is an important factor in the primary aggregation steps. Furthermore, valuable information about energetics, and, to some extent, structural features of the transition state, which defines the rate limiting aggregation step, might be obtained.

In the simplest case, this transition state corresponds to a bimolecular encounter complex. As shown in Figure 5 (left-hand blue bar), the calculated entropy difference implies a free energy difference between the monomers of system $\mathrm{H}$ and $\mathrm{HE}$ $\left(T \Delta S \sim 313 \mathrm{~K} \times 150 \mathrm{~J} \mathrm{~mol}^{-1} \mathrm{~K}^{-1}=47 \mathrm{~kJ} \mathrm{~mol}^{-1}\right)$; for the purposes of the illustration the enthalpy difference between the two systems is set to zero.

For comparison with the observed kinetics, the activation enthalpy difference $\Delta \Delta H^{+}=\Delta H_{\mathrm{HE}}^{\neq}-\Delta H_{\mathrm{H}}^{\neq}$between the two sys- 


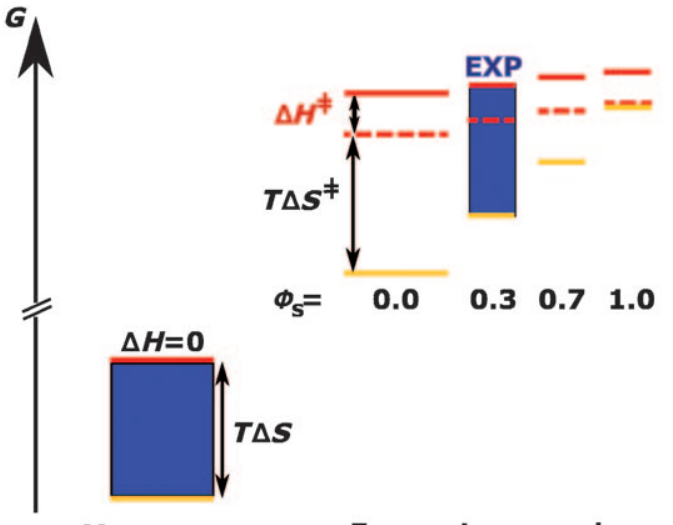

Monomers

Figure 5. Free energy profiles of the aggregation process for systems $\mathrm{H}$ (yellow) and HE (red). Free energies are based on entropy calculations for the monomers (left) and also include enthalpy estimates for the encounter complexes (right). The extent of the entropic contributions to the encounter complexes is characterized by the $\Phi_{\mathrm{S}}$ value. A $\Phi_{\mathrm{S}}$ value of 0.36 matches the measured difference in the free energy barriers (EXP). Note that deviating from the usual convention, $\Delta S^{\neq}=S_{H}^{\neq}-S_{H E}^{\neq}$denotes the difference between entropies at the barrier.

tems as well as the change in activation entropy $\Delta \Delta S^{+}=$ $\Delta S^{\neq}-\Delta S=\Delta S_{H}^{\neq}-\Delta S_{\mathrm{HE}}^{\neq}$need to be considered, which in Figure 5 appear at the encounter complex. Taking into account the overall excess charge of system $\mathrm{HE}(+4)$ and the neutrality of system $\mathrm{H}$, we assume $\Delta \Delta H^{+}$to be dominated by electrostatic repulsion between the HE monomers. We estimate this contribution of $14 \mathrm{~kJ} \mathrm{~mol}^{-1}$ from the electrostatic interactions between two solvated charged spheres at a distance of $2 \mathrm{~nm}$.

\section{$\Phi_{\mathrm{s}}$ values}

In contrast, $\Delta \Delta S^{\ddagger}$ cannot be readily estimated. Rather, $\Delta \Delta S^{\neq}$ depends on how much the flexibility of those "entropy-relevant" residues, which mainly contribute to the monomer entropy difference calculated above, is affected by the formation of the encounter complex. In analogy to the well-known $\Phi$ value, $^{[37]}$ which describes the energetic contribution of mutated amino acids to the transition state of a protein folding process, and thus yields structural information on the transition state, here we assign a $\Phi_{\mathrm{S}}$ value to the aggregation process at hand, $\Phi_{S}=\left(\Delta S-\Delta S^{\ddagger}\right) / \Delta S$. This value quantifies the extent to which the monomer entropy difference is affected in the encounter complex and should, therefore, provide structural information on the encounter complex.

Figure 5 illustrates the range of possible $\Phi_{\mathrm{s}}$ values. For $\Phi_{\mathrm{S}}=$ 0 , the monomer entropy difference is fully maintained at the barrier, so that the fluctuations of the entropy-relevant residues are unaffected by the formation of the encounter complex-most likely because they are not involved in the respective contact interfaces. In this case, the free energy barrier of system $\mathrm{HE}$ is increased with respect to the neutral system $\mathrm{H}$ only by the estimated enthalpic contribution of $14 \mathrm{~kJ} \mathrm{~mol}^{-1}$. This activation free energy difference, however, would imply that system $\mathrm{H}$ should aggregate approximately 200-times faster than system HE; this is not observed experimentally. If

the monomer entropy difference is not fully maintained $\left(\Phi_{\mathrm{S}}>\right.$ 0 ), the barrier heights of both systems would increase due to a correspondingly reduced entropy in the encounter complexes. Assuming that the entropy reduction is more pronounced for the more flexible system $\mathrm{H}$, its barrier height will increase faster; this implies a less pronounced difference in the aggregation kinetics of the two systems. For $\Phi_{\mathrm{S}}=1$, the entropy difference vanishes, and the activation free energy difference is $T \Delta S-\Delta H^{+}=33 \mathrm{~kJ} \mathrm{~mol}^{-1}$ (rightmost energy levels in Figure 5). In this case, system HE would display faster aggregation kinetics, in accordance with the experimental findings, albeit by orders of magnitude faster.

Since the three-times faster aggregation kinetics measured for system HE results in an activation free energy difference of $2.8 \mathrm{~kJ} \mathrm{~mol}^{-1}$, a $\Phi_{\mathrm{S}}$ value of 0.36 is obtained (Figure 5, righthand blue bar), and $64 \%$ of the entropy difference is maintained in the encounter complex. The corresponding partial reduction of the entropy difference from $47 \mathrm{~kJ} \mathrm{~mol}^{-1}$ in the monomeric systems to $30 \mathrm{~kJ} \mathrm{~mol}^{-1}$ in the encounter complexes suggests that only one of the two flexible regions identified above (C-terminal site or the helix of the B chain) is actually involved in contact formation. Considering that the two glutamates in the helix of the B chain are neutral in system $\mathrm{HE}$, which would enthalpically favor the formation of the encounter complex due to diminished local electrostatic repulsion, this region seems a likely candidate.

The discussion above considered the simplest case of a bimolecular encounter complex, which implies a very "early" transition state in the mechanism of fibril formation. Accounting for the polydispersity of the aggregation process on the pathway to fibrils the existence of intermediate oligomers cannot be ruled out. The observed correlation of the monomer decay with the formation of fibrils, however, renders an early transition state more likely. In any case, should the rate determining step-contrary to our expectation-correspond to a "late" transition state with an oligomeric complex structure, the discussion above would still apply, since entropy is also then expected to play an important, if not dominant role.

\section{Conclusions}

In summary, our combined study has revealed that the primary steps of $\mathrm{pH}$-dependent insulin aggregation are determined by the conformational flexibility of the monomer. The enthalpic and entropic contributions to the $\mathrm{pH}$-dependent activation free energy for aggregation have been quantified. Further, the observed correlation between the $\mathrm{pH}$-dependent monomer decay and fibril formation is consistent with an early rather than a late transition state in the insulin aggregation kinetics.

Contrary to what one might expect, the charged species (system HE) showed faster kinetics despite its enhanced solubility and electrostatic repulsion. This anomaly is explained in terms of an entropic overcompensation. Accordingly, compared to the charged monomers the neutral ones (system $\mathrm{H}$ ) display a larger flexibility, which is reduced in the encounter complex of the primary aggregation steps. This resulting entropic cost, which should be also present in an encounter com- 
plex rendering more an oligomeric rather than a bimolecular structure, increases the activation free energy barrier and thereby drastically slows down the aggregation kinetics.

The close relation of the $\mathrm{pH}$-dependence in the primary aggregation steps and entropy, which this study has revealed combined with the atomistic simulations, provides a microscopic picture and, in particular, allows the extraction of structural information on the encounter complex. Specifically, we suggest the residues in the helix of the $B$ chain to be part of the contact surface; this favors the idea that the interaction site of the encounter complex is structurally different from the dimer interface in the crystal structure. ${ }^{[20]}$

Indeed, a sequence of this helix (named the "steric zipper" motif involving residues B12-B17) was recently found to be crucial for the formation of amyloid-like fibrils and is incorporated in their cross- $\beta$ spines. ${ }^{[22,23]}$

\section{Experimental Section and Computational Methods}

Aggregation assays: Bovine insulin was purchased from Sigma-Aldrich (Product No. 15500) and was used without further purification. Hydrochloric acid $(1 \mathrm{~N})$ was purchased from Merck. Solvents were prepared by diluting the necessary amount of hydrochloric acid $(1 \mathrm{~N})$ with double distilled water to give the required $\mathrm{pH}$ (1.6, $2,2.5$ and 3) and were then filtered through a syringe filter $(0.45 \mu \mathrm{m}$ pore size). Insulin $(5.8 \mathrm{mg})$ was dissolved in the solvent of the required $\mathrm{pH}(20 \mathrm{~mL})$ to give a $50 \mu \mathrm{m}$ final solution. The $\mathrm{pH}$ values of the solvent and the final solution were verified to be within 0.1 of the desired value with a pH meter (Hanna Instruments). Aliquots $(1 \mathrm{~mL})$ were transferred into PP reaction vessels $(1.5 \mathrm{~mL})$ and shaken at $800 \mathrm{rpm}$ and $40^{\circ} \mathrm{C}$ in a block heater. Aliquots $\left(50 \mu \mathrm{L}\right.$ ) were sampled from the solution and stored at $-25^{\circ} \mathrm{C}$ for mass spectrometric analysis.

Mass spectrometry: The MS-based method for measuring the biokinetics of insulin in the present work was time-resolved liquid beam desorption mass spectrometry. ${ }^{[38-42]}$ The samples were introduced into the liquid water jet by using an HPLC injection valve (Rheodyne MX9925). For the laser desorption of protonated insulin the idler wave of a $\mathrm{LiNbO}_{3}$ optical parametric oszillator (OPO, GWU) with a wavelength of $2800 \mathrm{~nm}$ was employed. The OPO was pumped by the fundamental of a Nd:YAG laser (Spectra Physics, Quanta Ray INDI). Each injection resulted in 80 usable single shot spectra at $20 \mathrm{~Hz}$ repetition rate. The median average of the monomer peak intensities was calculated for further data analysis. The obtained traces (Figure 2A) usually consisted of several experiments (between 2 and 4), which were averaged in order to obtain a satisfactorily signal-to-noise ratio.

Atomic force microscopy: The solution containing the aggregation products was diluted by a factor of ten, $5-10 \mu \mathrm{L}$ of which were spin coated to dryness on a freshly cleaved mica surface. A Digital Instruments (Veeco) MultiMode scanning probe microscope Illa was used. Samples were imaged in air at a $1 \mathrm{~Hz}$ scan rate by using silicon tips.

Molecular dynamics simulations: The MD simulations with individual trajectory lengths of $95 \mathrm{~ns}$ were conducted with the GROMACS software package ${ }^{[43]}$ at $313 \mathrm{~K}$ by using the OPLS all-atom forcefield. ${ }^{[44,45]}$ Electrostatic interactions were calculated at every step with the particle mesh Ewald method. ${ }^{[46,47]}$ The starting structure for bovine insulin was obtained from the Protein Data Bank (PDB ID: 2BN3). Overlapping water atoms, alternate atoms with lower $B$ factors, as well as missing side chain atoms were corrected with the "FASTMD" command of the WHAT IF modeling software. ${ }^{[48]}$ The MEAD package was used to calculate $\mathrm{p} K_{\mathrm{a}}$ values ${ }^{[31,49]}$ (see the Supporting Information). Each simulation box contained the protein monomer solvated in explicit water (TIP4P) ${ }_{i}^{[50]}$ sodium or chloride ions were added to achieve a zero net charge of the system. The integration time step was chosen to be $1 \mathrm{fs}$ and weak coupling algorithms were applied to the system over the course of the simulations to keep the temperature and pressure constant. All analyses were performed over the last $75 \mathrm{~ns}$ of each trajectory to exclude initial relaxation motions. The $C_{\alpha}$ atoms were used for all principal component analysis. From a principal component analysis over the combined trajectories, after assembling and diagonalising the covariance matrix of atom pairs, the eigenvectors and eigenvalues were obtained. VMD was used for all 3D structure plots. ${ }^{[51]}$

\section{Acknowledgements}

We thank Bert L. de Groot and Gerrit Groenhof for carefully reading the manuscript, Thomas Jovin for helpful discussions, and the Deutsche Volkswagenstiftung (grants 1/80436, 1/80585), the Sonderforschungsbereich 755, and the Graduate School 782 of the German Science Foundation (DFG) for financial support.

Keywords: aggregation kinetics · entropy · insulin · mass spectrometry $\cdot$ molecular dynamics

[1] P. Halban, Diabetologia 1991, 34, 767-778.

[2] C. Bryant, D. Spencer, A. Miller, D. Bakaysa, K. McCune, S. Maple, A. Pekar, D. Brems, Biochemistry 1993, 32, 8075-8082.

[3] G. Dodson, D. Steiner, Curr. Opin. Struct. Biol. 1998, 8, 189-194.

[4] F. E. Dische, C. Wernstedt, G. T. Westermark, P. Westermark, M. B. Pepys, J. A. Rennie, S. G. Gilbey, P. J. Watkins, Diabetologia 1988, 31, 158-161.

[5] J. Brange, G. Dodson, D. Edwards, P. Holden, J. Whittingham, Proteins Struct. Funct. Gen. 1997, 27, 507-516.

[6] J. Brange, L. Langkjsgmaelig, S. Havelund, A. Volund, Pharm. Res. 1992, 9, 715-726

[7] J. Brange, S. Havelund, P. Hougaard, Pharm. Res. 1992, 9, 727-734.

[8] C. M. Dobson, Nature 2003, 426, 884-890.

[9] W. Wang, Int. J. Pharm. 2005, 289, 1-30.

[10] J. Jimenez, E. Nettleton, M. Bouchard, C. Robinson, C. Dobson, H. Saibil, Proc. Natl. Acad. Sci. USA 2002, 99, 9196.

[11] C.-C. Lee, A. Nayak, A. Sethuraman, G. Belfort, G. J. McRae, Biophys. J. 2007, 92, 3448-3458.

[12] Q. Hua, M. Weiss, J. Biol. Chem. 2004, 279, 21449-21460.

[13] M. J. Burke, M. A. Rougvie, Biochemistry 1972, 11, 2435-2439.

[14] Q. Hua, S. Shoelson, M. Kochoyan, M. Weiss, Nature 1991, 354, 238-241.

[15] A. Kline, R. Justice, Jr., Biochemistry 1990, 29, 2906-2913.

[16] T. P. J. Knowles, W. Shu, G. L. Devlin, S. Meehan, S. Auer, C. M. Dobson, M. E. Welland, Proc. Natl. Acad. Sci. USA 2007, 104, 10016-10021.

[17] A. Ahmad, V. N. Uversky, D. Hong, A. L. Fink, J. Biol. Chem. 2005, 280, 42669-42675

[18] R. Jansen, W. Dzwolak, R. Winter, Biophys. J. 2005, 88, 1344-1353.

[19] D. F. Waugh, J. Am. Chem. Soc. 1946, 68, 247-250.

[20] L. Nielsen, S. Frokjaer, J. Brange, V. Uversky, A. Fink, Biochemistry 2001 40, 8397-8409.

[21] L. Nielsen, R. Khurana, A. Coats, S. Frokjaer, J. Brange, S. Vyas, V. Uversky, A. Fink, Biochemistry 2001, 40, 6036-6046.

[22] M. I. Ivanova, M. J. Thompson, D. Eisenberg, Proc. Natl. Acad. Sci. USA 2006, 103, 4079-4082.

[23] M. R. Sawaya, Nature 2007, 447, 453-457. 
[24] C. Dobson, M. Karplus, Curr. Opin. Struct. Biol. 1999, 9, 92-101.

[25] V. Zoete, M. Meuwly, M. Karplus, Proteins Struct. Funct. Bioinf. 2005, 61, 79-93.

[26] J. L. Whittingham, D. J. Scott, K. Chance, A. Wilson, J. Finch, J. Brange, G. G. Dodson, J. Mol. Biol. 2002, 318, 479-490.

[27] B. Tidor, M. Karplus, J. Mol. Biol. 1994, 238, 405-414.

[28] J. T. Jarrett, P. Lansbury, Cell 1993, 73, 1055-1056.

[29] J. W. Kelly, Nat. Struct. Mol. Biol. 2000, 7, 824-826.

[30] A. Arora, C. Ha, C. B. Park, Protein Sci. 2004, 13, 2429-2436.

[31] D. Bashford, K. Gerwert, J. Mol. Biol. 1992, 224, 473-486.

[32] V. Zoete, M. Meuwly, M. Karplus, J. Mol. Biol. 2004, 342, 913-929.

[33] O. F. Lange, H. Grubmüller, Prot. Struct. Funct. Bioinf. 2006, 62, 10531061.

[34] O. F. Lange, U. Hensen, H. Grubmüller, Phys. Rev. E 2009, in press.

[35] J. Schlitter, Chem. Phys. Lett. 1993, 215, 617-621.

[36] D. C. Sullivan, C. M. Lim, J. Chin. Chem. Soc. 2004, 51, 1209-1219.

[37] A. Fersht, A. Matouschek, L. Serrano, J. Mol. Biol. 1992, 224, 771-782.

[38] A. Charvat, A. Boegehold, B. Abel, Aust. J. Chem. 2006, 59, 81-103.

[39] A. Charvat, B. Abel, Phys. Chem. Chem. Phys. 2007, 9, 3335-3360.

[40] A. Charvat, E. Lugovoj, M. Faubel, B. Abel, Eur. Phys. J. D 2002, 20, 573582.

[41] B. Abel, A. Charvat, U. Diederichsen, M. Faubel, B. Girmann, J. Niemeyer, A. Zeeck, Int. J. Mass Spectrom. 2005, 243, 177-188.
[42] W. Kleinekofort, J. Avdiev, B. Brutschy, Int. J. Mass. Spectr. Ion Proc. 1996, $152,135-142$

[43] D. van der Spoel, E. Lindahl, B. Hess, G. Groenhof, A. E. Mark, H. J. C. Berendsen, J. Comput. Chem. 2005, 26, 1701-1718.

[44] W. Jorgensen, D. Maxwell, J. Tirado-Rives, J. Am. Chem. Soc. 1996, 118 $11225-11236$

[45] G. Kaminski, R. Friesner, J. Tirado-Rives, W. Jorgensen, J. Phys. Chem. B 2001, 105, 6474-6487.

[46] U. Essmann, L. Perera, M. L. Berkowitz, T. Darden, H. Lee, L. G. Pedersen, J. Chem. Phys. 1995, 103, 8577-8593.

[47] T. Darden, D. York, L. Pedersen, J. Chem. Phys. 1993, 98, 10089-10092.

[48] G. Vriend, J. Molec. Graphics 1990, 8, 52-56.

[49] "Scientific Computing in Object-Oriented Parallel Environments: First International Conference", D. Bashford, Proceedings ISCOPE 97, Marina Del Rey, California, December 8-11, 1997.

[50] W. L. Jorgensen, J. Chandrasekhar, J. D. Madura, R. W. Impey, M. L. Klein, J. Chem. Phys. 1983, 79, 926-935.

[51] W. Humphrey, A. Dalke, K. Schulten, J. Molec. Graphics 1996, 14, 33-38.

Received: April 29, 2009

Published online on June 16, 2009 